\title{
New Method of Transmission Electron Diffraction to Characterize Nanomaterials in the SEM*
}

\author{
Roy H. Geiss ${ }^{1,3}$, Robert Keller ${ }^{1}$, Scott Sitzman $^{2}$, Paul Rice ${ }^{3}$
}

1. National Institute of Standards and Technology, 325 Broadway, Boulder CO 80305

2. Oxford Instruments America, Concord MA 01742

3. Nanomaterials Characterization Facility, University of Colorado, Boulder CO 80309

A new technique of electron diffraction for obtaining the crystallographic characteristics of nanoparticles, nanowires and thin films with dimensions down to $10 \mathrm{~nm}$ using scanning electron microscopy is described. This technique, denoted as transmission Electron Forward-Scatter Diffraction (t-EFSD), involves the capture of the spatial variation in large-angle forward scattering of electrons in transmission, using commercially available electron backscatter diffraction (EBSD) detectors and software. Improvements in both diffraction pattern quality and spatial resolution were achieved, compared to conventional EBSD.

In conventional EBSD a pattern is acquired by imaging the spatial variation in coherent electron backscattering from a specimen surface that is tilted towards a position-sensitive detector, with the specimen normal typically at an angle of approximately $70^{\circ}$ away from perpendicular to the optic axis of the microscope. In this geometry the primary beam scatters incoherently and slightly inelastically within the crystal. This results in a new internal divergent electron source that subsequently gives rise to coherently scattered electrons that form the resulting Kikuchi diffraction pattern. Simulations using dynamical electron diffraction theory demonstrate that the intensities in the patterns are determined largely by energy absorption effects on electrons that scatter out of the coherent Bloch wave field. From a practical standpoint, factors such as degree of local surface roughness or crystal perfection play an important role in determining whether observable Kikuchi bands are detected. Acquisition of good quality patterns from extremely fine particles or thin films on substrates can be difficult when the specimen dimensions are smaller than the backscattered electron interaction volume. However, it is possible to obtain patterns from specimens such as thin foils or nanoparticles when the effect of diffuse scattering from thick mounting substrates has been reduced as will be described here. Additionally, such patterns usually have higher quality than those obtained from bulk specimens.

The work presented here is based on the forward-dominance of inelastic scattering inherent in a SEM. The sample, which must be transparent to electrons, is oriented approximately $90^{\circ}$ from that described above and conventional EBSD detection hardware is used to collect the resulting Kikuchi patterns. We refer to the phenomenon as transmission Electron Forward Scatter Diffraction (t-EFSD). The data presented here were all collected by use of a commercial EBSD system mounted on a variety of SEMs with either Schottky or W-emitters. All specimens were either TEM thin foils or mounted on TEM grids. The microscopes were operated at $15 \mathrm{kV}$ to $30 \mathrm{kV}$ with probe currents greater than $300 \mathrm{pA}$ and at working distances of $3 \mathrm{~mm}$ to $12 \mathrm{~mm}$. Monte Carlo scattering simulations demonstrate the effects of both the transmission geometry and the tilt of the specimen surface normal away from the detector, with emphasis on differences in signal energy and probe size at the specimen exit surface. The simulations showed that electrons detected in the transmission geometry exhibit both higher average energy and narrower energy spread than electrons collected with the conventional backscatter geometry. Examples of the application of t-EFSD are given in figures 1 to 4 . Figure 1 demonstrates t-EFSD applied to a $40 \mathrm{~nm}$ film of $\mathrm{Ni}$ on a $\mathrm{Si}_{3} \mathrm{~N}_{4}$ substrate. Figure 2 shows t-EFSD obtained from $15 \mathrm{~nm}$ particles of Fe-Co. Figure 3 shows high quality patterns using t-EFSD from GaAs nanowires. Figure 4 shows t-EFSD inverse pole figure map and patterns obtained from a TEM type sample of an electropolished foil of $\mathrm{Cu}$.

* Contribution of the U.S. Department of Commerce, NIST. Not subject to copyright in the U.S.A 

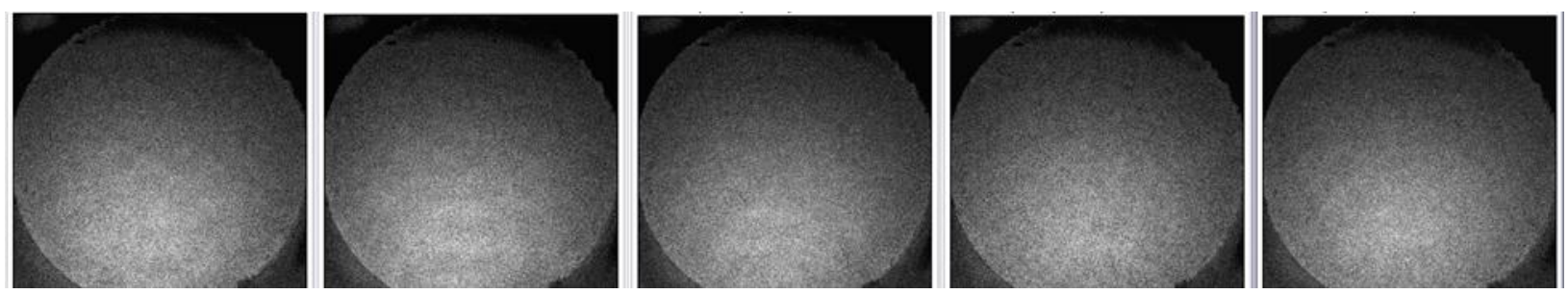

Figure 1a. $40 \mathrm{~nm}$ thick $\mathrm{Ni}$ film on $\mathrm{Si}_{3} \mathrm{~N}_{4}$ substrate with data taken in the conventional EBSD reflection orientation. Patterns are difficult or impossible to index. Ni grain size $<40 \mathrm{~nm}$.
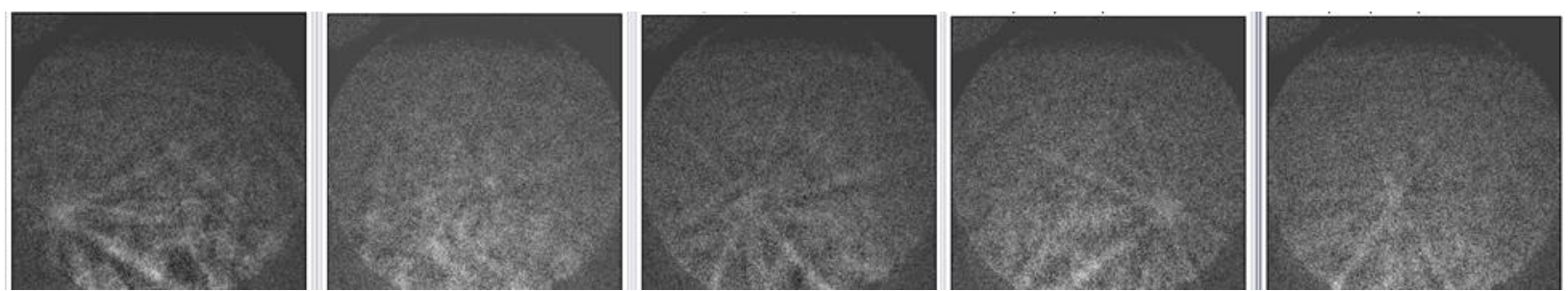

Figure 1b. $40 \mathrm{~nm}$ thick $\mathrm{Ni}$ film on $\mathrm{Si}_{3} \mathrm{~N}_{4}$ substrate with data taken in t-EFSD mode, with Ni side down in the orientation for transmission diffraction. Patterns can all be indexed easily.

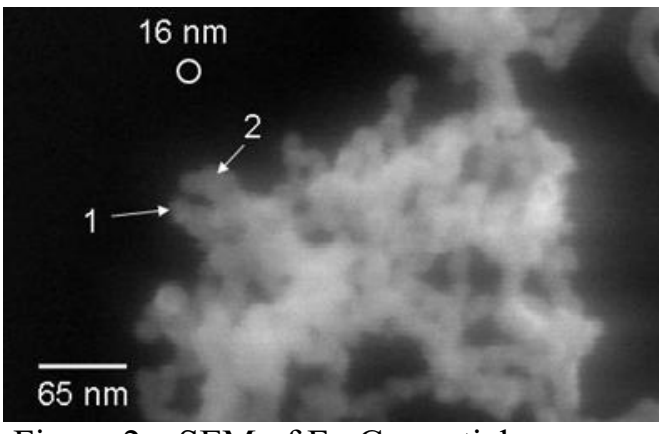

Figure 2a. SEM of Fe-Co particles.

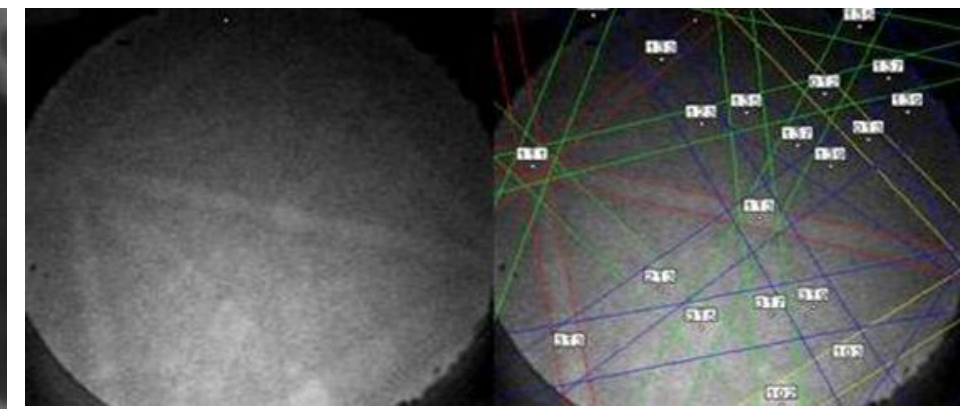

Figure 2 b. t-EFSD pattern and index off particle 1 in fig. $2 a$.

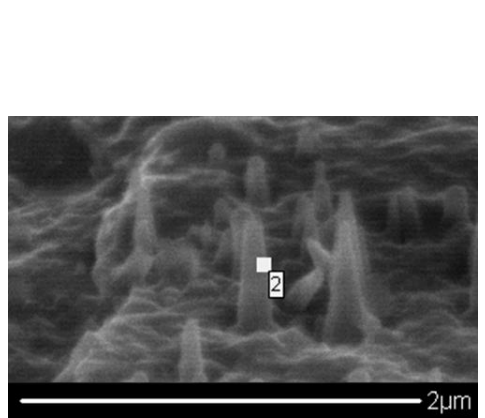

Fig. 3a. SEM of GaAs wires.

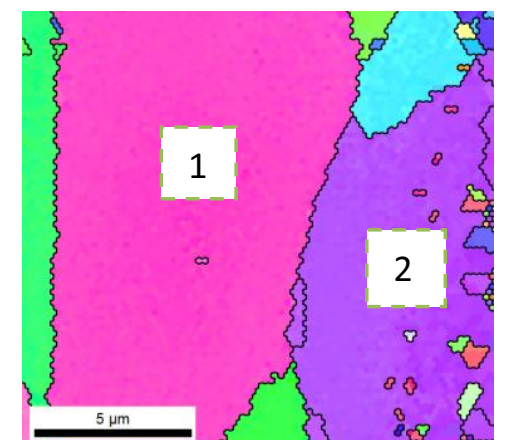

Fig. 4a. t-EFSD map off $\mathrm{Cu}$ foil.
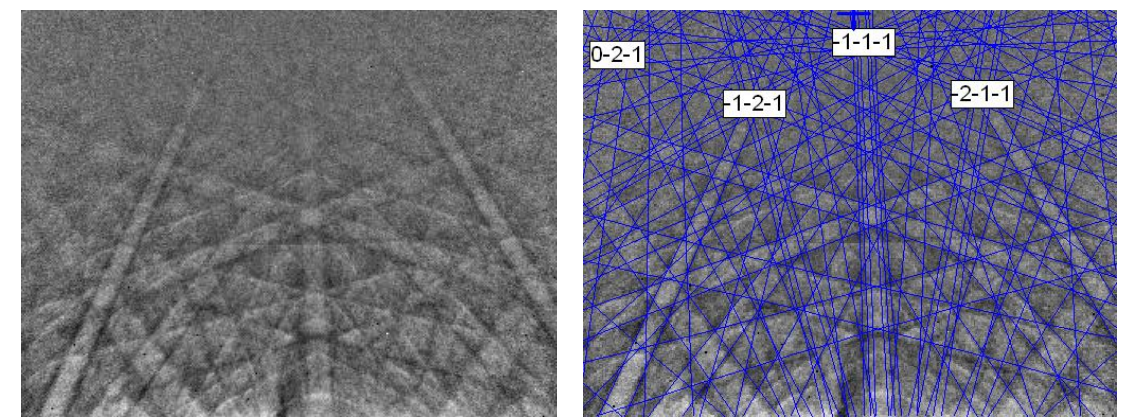

Fig. 3b. t-EFSD from pt. 2 in fig. 3a. Fig. 3c. Indexed pattern of fig. 3b.

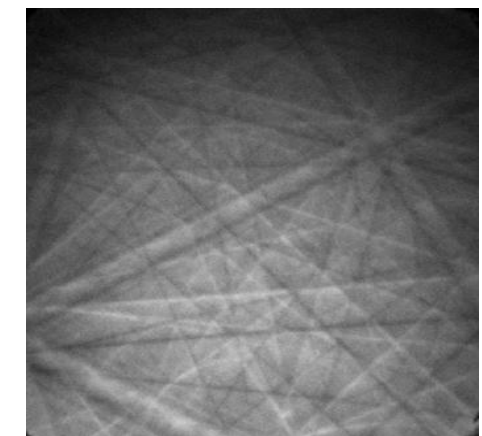

Fig. 4b. t-EFSD from grain 1.

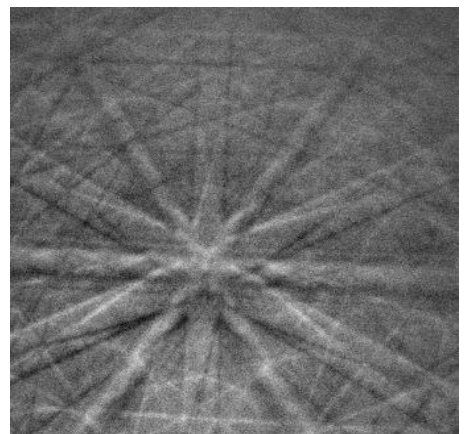

Fig. 4c. t-EFSD from grain 2. 